\title{
Enabling Information Sharing in a Port
}

\author{
Peter Bjerg Olesen*, Hans-Henrik Hvolby, and Iskra Dukovska-Popovska \\ Centre for Logistics, Department of Mechanical and Manufacturing Engineering, \\ Aalborg University, Denmark \\ pbo@m-tech.aau. dk
}

\begin{abstract}
Ports are integral parts of many supply chains and are as such a contributing factor to the overall efficiency of the supply chain. As the role of the ports is increasingly changing towards being more integrated into the supply chain, ports need to start focusing on optimising the activities and striving for low-lead time. Ports are also complex entities comprising of different companies. The dynamic nature of ports is also a problem when trying to optimise the utilisation of resources and ensure a low lead-time. Information sharing is crucial in the attempt to improve ports operations. This paper attempts to explain how information sharing is enabled in such an environment, and which considerations are relevant, both in regards to the information and required technology. The paper highlights trust, availability of data, and complexity of solutions and technology, as being the main hurdles.
\end{abstract}

Keywords: Information sharing, technology, ICT, information flow, port.

\section{Introduction}

Port systems are an important part of many supply chains, and an important contributor to the overall (in)efficiency of a supply chain. As the focus on supply chain efficiency has increased in previous years, the focus on the efficiency of ports is also increasing. The activities in a port are directly dependant on the input from external parties, both hinterland (the geographical inland area the port services) and foreland (the customers sending and receiving goods through the port outside the hinterland).

The amount and time of transactions going to and from hinterland can be quite uncertain, which affects the planning and control of capacity and activities at a port. The performance of a port terminal, can suffer greatly from this uncertainty in terms of lead-time and bottle-necks i.e. the Forrester effect [1]. Therefore the uncertainty needs to be minimised. In order to plan the capacity better, it is necessary to get input from ships arriving, as well as information from transportation companies on arrival of trucks.

The performance of a production unit, e.g. a port terminal, can suffer greatly from this uncertainty in terms of lead-time and bottle-necks i.e. the Forrester effect [1]. Therefore the uncertainty needs to be minimised. To reduce these uncertainties there

\footnotetext{
* Corresponding author. 
two general methods: First; reduce the uncertainty factors as seen in Lean production when levelling demand and supply. This is done by having more knowledge about your customers and suppliers activities. Second; be more responsive by communicating better between the partners in the supply chain so any changes are communicated as fast as possible, giving supply chain partners the chance to react to the changes [2]. This paper will explain how information sharing can be enabled in a port environment, and which considerations are relevant, both in regards to the information and required technology. Furthermore, the paper highlights the main obstacles for implementation.

To define information sharing as a possible solution to a problem, some of the benefits are introduced; hereafter the prerequisites for enabling information sharing are discussed. A case study is introduced to exemplify the problems identified towards implementing information sharing. As a first step to solve the lack of sharing, some of the general requirements are presented.

\section{Information Sharing and Its Benefits}

Bichou et al [3] describe the lack of close collaboration among supply chain members in the port and shipping industry and encourage ports to be more proactive in their role to increase collaboration. Further, Robinson [4] encourages ports to become active elements in the value driven supply chain.

The general level of supply chain integration and information sharing at small ports has not developed much, even though the benefits have been thoroughly described in the literature. Lee et al [5], Lee et al [6] and Flynn et al [7] show the positive effect of information sharing in the supply chain in general, but how to share the information is not thoroughly analysed. Even though technical solutions, for many years, have supported exchange of data, they have not been widely applied. According to Braziotis et al [8] one reason is that many companies focus on short term profit, which has kept them from building long term strategic partnerships in the supply chain. Also the fear for indirectly providing competitors with sensitive data has been main barrier. Further the complexity of some of the solutions has also been too high, as for small and medium sized companies to adapt them. This means there is a possibility in creating a form of data sharing that takes small/medium sized companies into account.

Zhou et al [9] shows how information sharing benefits supply chain practices in dynamic supply chain environments, by reducing the amount of unknown factors, increasing the ability of a company to plan their own operations in synchronisation with the rest of the supply chain. Based on the study done by Zhou et al [9], the effectiveness of information sharing as a means of performance enhancing, depends on the level of dynamism in the supply chain. This means that in supply chains where demand is very stable, extra information sharing has no performance enhancing effect. However, it is shown that when demand is unstable it can be essential to have an effective information sharing system, in order to receive important information as fast as possible, allowing companies to adjust capacity before it is committed.

One problem is the lack of information exchange in the dynamic port environment, in terms of having the most correct information when planning. The main causes leading to this problem have been identified as follows: 
- Trust, [8]

- Availability of data, Quality of data [10]

- Complexity of system/technology

The trust issue is a major obstacle for information sharing as it is the basis of collaborating with anyone. In normal supply chain setups it is important to have trust, but in a port environment where there are a lot of shared resources it is even more important, as data can cross between users of the same resources. The information sharing solution should therefore focus on trying to circumvent the trust issue, by allowing companies to share according to their comfort level. Trust as a psychological phenomenon is outside the scope of this paper.

\section{System Requirements for Enabling Information Sharing}

According to a literature review by Perego et al [11], there is a lack of focus in the research on ICT solutions for transport companies, how they should operate and communicate with partners. However, in the literature, ICT is often named as a solution without providing information or analysis of how the ICT system should work in order to support certain functionality. This is a problem when trying to actually create an information sharing setup, as there no description of how to do this, and especially for smaller economic scale supply chain actors, such as a small/medium sized port, this is also confirmed by Robinson [4], especially in regards to implementing ICT solution.

For the small SC actors to involve in an exchange of information, it is important to find a way that is simple and not too expensive. Based on literature, three methods that comply with these criteria have been identified:

- Manual information sharing

- Phone calls, meetings, email, spread sheets etc.

- Kia et al [10] discuss reducing manual efforts in 2000, little has happened.

- Database access (ERP or similar ICT system)

- Direct link to a partners ERP database, to read/write in real-time. Allow realtime transaction knowledge as well as the possibility to view order-lines etc.

- Not useable due to the trust issues and security issues with remote access to central company systems.

- Dedicated ICT system for information sharing

- Portal placed between partners allowing for easy database integration and permission configuration.

- Keceli [12] presents an information model for a port, based on a centralistic system.

The third suggested method can provide new functionality, which has the possibility of innovating the information exchange climate, and does not pose trust and security issues directly. The two other methods have proven not to be the most effective solutions. 
A system that enables information sharing should be easy to use and to add/extract information. Further it should handle the information exchange automatically, when signed up, and in real time. The updated information could help organisations adjust their plan to a more optimal order, e.g. before execution of an order line. Stefansson et al [13] have defined certain types of information that are essential to supply chain interactions, and therefor relevant for inclusion in such a system. These criteria should be used when deciding what to share.

The information sharing system should be trust neutral, so the information in the system is only there, because companies have decided to share the information and with whom to share it. This requires the system to provide easy data sharing configuration, with the option of managing each partner alone or as a group. The system will also have to support any ERP or other resource management systems. This would be obtained by configuring the integration in every installation. And as long as the data is available in the company's database it should be a question of pointing the sharing system to this location.

A voluntary system is not by any means a complete solution to the issue of uncertainty in demand and supply for a port. It is however an option for smaller supply chain partners as they will have the possibility to integrate information with others, without having to force a certain system to be used by all partners, as seen with large corporations e.g. Walmart. Therefore the system should not be dependent on every partner using it. It should be seen as an addition to traditional information sources.

With the description of what the system should be able to do, the next phase is to present a port context and the need for information sharing.

\section{Case Study}

The case port is a small to medium size port with around 1800 ship berths a year. The main traffic is oil, bulk, containers and specialised cargo.

In the port there are seven main actors:

- Port system

- Port authority

- Port Terminal

- Stevedore
- Transporters

- Shipping lines

- Truck carriers
- Customer

- Shipping brokers

- Direct customers

The port authority handles docking permission and other things regarding pre docking activities as well as some services for the ships such as fresh water. The port terminal handles the goods and moves it from ship to truck or vice versa. The stevedore is the dockworkers handling some of the manual operation required in connection with loading and unloading. There are two types of transporters: one going to and from the hinterland, and another going to and from the foreland, here represented by trucks and ships. The final part of the port system is the customer making an order to have goods transported through the port, often represented by a shipping broker, who makes the transport bookings for the customer. 
In this port, seven different actors are in possession of information that relates to the transport of e.g. a container. The terminal, which is the focus of the paper, does the physical movement of goods from one transport form to another. Currently, the terminal only receives information about when the goods arrive at the terminal. Ship arrivals is the only information the terminal has in advance, as the ship arrivals follow a calendar, but delays in ship arrival can cause problems as the arrival time is not monitored in real-time. All planning of capacity (docks, cranes, people) is done around the arrival of ships. When it comes to trucks delivering or picking up goods, there is no planning done at the terminal. It is known that trucks tend to arrive around the departure of the ship, but the order of trucks is unknown. The information exchange that does exist today between the operators (cranes, trucks, etc.) is done by phone and email, which results in the information sometimes is delayed, forgotten or simply not communicated.

The consequence of the missing information can be seen in trucks waiting at the gate and waiting to be serviced by the terminal operators. Every time there is a ship arrival, many truck carrier firms and customers tend to wait until latest possible time to deliver the goods to the terminal. The effect of this is that the terminal is overburdened at these peak periods, resulting with long lead time, and is equally unburdened in the remaining timeframe, i.e. low resource utilisation. These uncertainties also lead to frequent use of overtime for the workers, as well as lot of waiting time.

To remedy these issues for the case port, the concept of the information sharing setup is used to describe a future state scenario.

\section{System Framework}

Two things are considered when describe the model for the information sharing system: 1) information flow model and 2) technology considerations. To actually create and implement an information sharing system a lot of other aspects are also necessary, such as system interoperability, however this is not a scope of this paper.

\subsection{Information Flow}

This paper will focus on information that relates to capacity use, such as delivering a container or a ship docking and the processes that are tied to this activity, such as reach stackers picking and moving containers.

The information in a port system can be segmented into two types: first, order information that sets up the overall flow through the port, and secondly, real-time information that enables the capacity to be adjusted until a firming deadline. Because of the simplistic approach to real-time data the information needed for reducing dynamism is also reduced in complexity. According to Stefansson et al [13] it is value adding information that should be shared, and defining what information is value adding depends on the reference point. The terminal is the reference in this paper; therefore the information needed is arrival and departure time and dimensions of cargo. For a container the information needed is: size, weight, destination, departure and arrival time. 
With this information the container terminal will be able to plan the containers route through the terminal. This can be seen in a simple general model of the information flow in a port, Fig. 1.

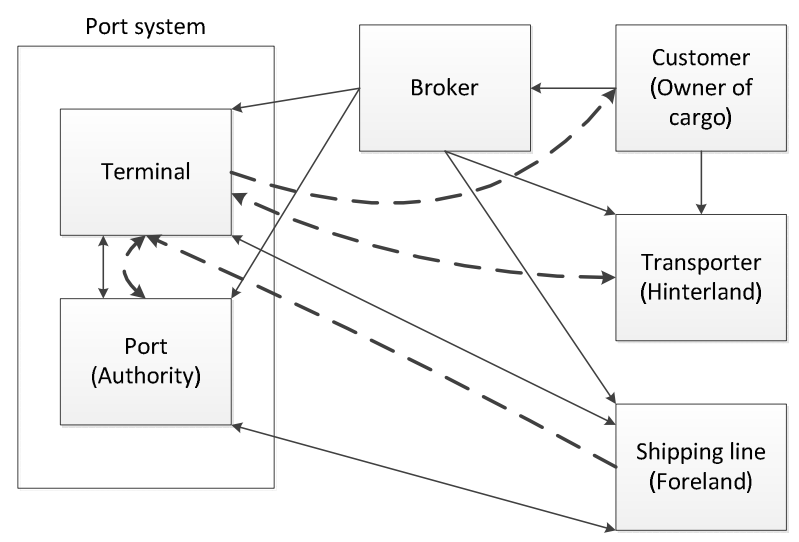

Fig. 1. Order flow and real-time information flow. Dotted line is the real-time information

The real-time information needed is related to the terminal as this is the focal point. The terminal communicates arrivals of goods to customers and transporters, and transporter and shipping line communicate to the terminal.

The point of Fig. 1 is to illustrate that the requirements for the information exchange system can be very simple, and only a limited subset of information needs to be shared with partners dynamically, decreasing the influence of the trust factor and the complexity. The dotted line represents the information that will benefit from being transmitted real-time, such as time and dimension information. The solid lines represent the existing flow of sales and purchase orders; this then needs to be supplemented with an information update scheme.

In terms of availability and quality of data, the reduced amount of real-time data exchange in this suggestion ensures that the resources needed for making and maintaining this information is kept at a minimum.

Lastly the complexity of the information flow is lower as opposed to a system that keeps track of all data, not just data that is changing. Further, the lower complexity of information ensures that the people working with the information can utilize it better and thus making improvements based on it. These assumptions are based on small companies without data specialists to understand and connect incoming data to their own system.

\subsection{Technology Considerations}

To enable the information sharing it is also necessary to have an IT system. This section will address technological considerations required for such a system. There are four aspects to technology in the scope of this paper: trust, availability, complexity and cost. 
- The trust parameter is important to many companies, as they need to know and get comfortable with how the technology works. Further trust is also dependent on the security of the technology, and whether it can make the company vulnerable.

- The availability is the first parameter as a company evaluate its requirements specification.

- Is the needed functionality available in existing technology?

- If not revise the functionality demand or pay for development.

- The complexity is perhaps the most important parameter as too high complexity of technology is the most common cause of failures in technology projects. The complexity can be viewed in several ways.

- The size and functionality sample of the system.

- Bad support or poor documentation increases complexity.

- Last is the cost, as this decides if the technology projects are within an economical margin and is the requested specification expensive in terms of the systems possible benefits, e.g. purchasing an ERP with all modules, or instead purchase a smaller more basic ERP system that fits better with economy and complexity.

These considerations make it possible to find define the real needs for an information sharing system in small/medium sized port environment. The main focus is on trust, availability, complexity and cost of information and technology used to provide information to the port systems actors.

\section{Results and Discussion}

This paper highlights the benefits of information sharing, where dynamic environments have the largest benefits, as an increased information flow improves the responsiveness of the entire system. However, there where perhaps a lack in the literature on how this information sharing should be facilitated.

To enable information sharing there are a lot of considerations to be done, in this paper it is limited to information flow and technology considerations, as these are the first step in order to make a model of a complete information sharing system, including technical setup. The technology available today will support all required functions in some way, but in a complex and costly manner. So to find more optimal solutions, a sharing system has to adapt to the reality of the system it has to support.

The system should be easy to use and be a used on a voluntary basis, and not be dependent on every partner using the system, but an addition to traditional information sources.

The implications to research are an increased focus on the difficulties and possibilities of information sharing in small ports. Through further work the need for manual interfaces between supply chain partners should be reduced, and allow the possibilities of real-time information and adjustments.

The proposed approach does in itself not provide much in terms of performance improvement, but it builds a foundation that would allow for better coordination between supply chain partners. This would reduce waiting times, and improve resource utilisation by smoothing the demand. 


\section{References}

1. Lee, H.L., Padmanabhan, V., Whang, S.: Information distortion in a supply chain: The bullwhip effect. Management Science 43, 546-558 (1997)

2. Christopher, M.: The Agile Supply Chain: Competing in Volatile Markets. Industrial Marketing Management 29, 37-44 (2000)

3. Bichou, K., Gray, R.: A logistics and supply chain management approach to port performance measurement. Maritime Policy \& Management: The Flagship Journal of International Shipping and Port Research 31, 47 (2004)

4. Robinson, R.: Ports as elements in value-driven chain systems: the new paradigm. Maritime Policy \& Management 29, 241-255 (2002)

5. Lee, H.L., So, K.C., Tang, C.S.: The Value of Information Sharing in a Two-Level Supply Chain. Management Science 46, 626-643 (2000)

6. Lee, H.L., Whang, S.: Information sharing in a supply chain. International Journal of Manufacturing Technology and Management 1, 79-93 (2000)

7. Flynn, B.B., Huo, B., Zhao, X.: The impact of supply chain integration on performance: A contingency and configuration approach. Journal of Operations Management 28, 58-71 (2010)

8. Braziotis, C., Tannock, J.D.T.: Building the Extended Enterprise: Key Collaboration Factors. The International Journal of Logistics Management 22, 4-4 (2011)

9. Zhou, H., Benton Jr., W.C.: Supply chain practice and information sharing. Journal of Operations Management 25, 1348-1365 (2007)

10. Kia, M., Shayan, E., Ghotb, F.: The importance of information technology in port terminal operations. International Journal of Physical Distribution \& Logistics Management 30, 331-344 (2000)

11. Perego, A., Perotti, S., Mangiaracina, R.: ICT for logistics and freight trans-portation: a literature review and research agenda. International Journal of Physical Distribution \& Logistics Management 41, 457-483 (2011)

12. Keceli, Y.: A proposed innovation strategy for Turkish port administration policy via information technology. Maritime Policy \& Management 38, 151-167 (2011)

13. Stefansson, G., Lumsden, K., Mirzabeiki, V.: Smart Transportation Management Systems to Support Visibility of the Supply Chain Information Types. Presented at the 16th ITS World Congress and Exhibition on Intelligent Transport Systems and Services (2009) 